\title{
Precision Medicine and Vaccination of Older Adults: From Reactive to Proactive (A Mini-Review)
}

\author{
T. Mark Doherty ${ }^{a} \quad$ Alberta Di Pasquale ${ }^{a} \quad$ Jean-Pierre Michel ${ }^{b}$ \\ Giuseppe Del Giudice ${ }^{c}$ \\ ${ }^{a}$ Global Medical Affairs, GSK, Wavre, Belgium; ${ }^{b}$ Geriatric Department, University of Geneva, Geneva, Switzerland; \\ 'Vaccine Research and Development Discovery Performance, GSK, Siena, Italy
}

\section{Key Messages}

- As populations age, the total disease burden due to non-communicable or chronic conditions in older adults increases: infectious diseases are a significant and under-appreciated contributor to poor outcomes in this population.

- Vaccination has been shown to dramatically improve outcomes in patients with chronic comorbidities but is almost always under-used in adults.

- If vaccination programs for adults reached their full potential, the risk of hospitalization or death due to both infectious and non-infectious diseases could be effectively lowered.

- A precision medicine approach, which integrates vaccination as part of a package of health interventions targeting an individual's needs, may offer a promising complementary approach to improving coverage and reducing the total burden of disease in addition to current public health interventions.

\section{Keywords}

Precision medicine $\cdot$ Vaccination $\cdot$ Non-communicable diseases $\cdot$ Older adults

\begin{abstract}
As populations age globally, the health of older adults is looming larger on the agendas of public health bodies. In particular, the priority is to ensure that older adults remain healthy, independent, and engaged in their communities. In other words, ensuring that increasing life spans are matched by increasing "health spans," meaning years spent in good health. Chronic conditions such as cancer or respiratory and cardiovascular diseases account for the bulk of the disease
\end{abstract}

karger@karger.com www.karger.com/ger

Karger!

\section{(C) 2019 The Author(s)}

Published by S. Karger AG, Basel

Karger

0 pen access

This article is licensed under the Creative Commons Attribution 4.0 International License (CC BY) (http://www.karger.com/Services/ OpenAccessLicense). Usage, derivative works and distribution are permitted provided that proper credit is given to the author and the original publisher. burden in older adults, and the consensus is that these can best be tackled by effective primary prevention. However, given the diverse nature of older populations, whose prior health experiences can be complicated by multi-morbidity and poly-pharmacy, effective primary prevention can be challenging. One approach that is gaining momentum is what is called "precision" or P4 medicine. The acronym stands for "predictive, personalized, preventive, participatory" medicine, and is based on the premise that preventing disease is better than treating it. However, effective prevention requires the ability to predict disease risk for a given patient, the tailoring of treatment to their circumstances, and their consent for or participation in the offered treatment. A P4 approach may seem counter-intuitive, given that
Giuseppe Del Giudice

Translational Science, R\&D Center Italy, GSK

Via Fiorentina 1

IT-53100 Siena (Italy)

E-Mail giuseppe.x.del-giudice@gsk.com 
vaccination is generally considered a public health intervention. However, in this article, we discuss the application of P4 medicine as a complement to planning the vaccination of older individuals, with a special focus on the important role that vaccine-preventable infections play in the burden of non-communicable disease. @2019 The Author(s)

Published by S. Karger AG, Basel

\section{Introduction}

In a little over a century there has been a dramatic increase in human life expectancy, driven by improved living conditions, better medical care, and a reduced risk of premature death [1]. As a result, the global population is ageing rapidly, with increases in both the proportion and the absolute number of older people [2]. As a result, the global increase in life expectancy, though still cause for celebration, has brought about a corresponding change in anxieties. People may worry less about dying prematurely of infectious disease, but more about the risk of a prolonged period of ill-health in old age. These worries encompass both the threat of lost health and autonomy and the prospect of the financial costs associated with chronic ill-health. The potential financial costs of an aging population are also of concern to health care providers, pension funds, and governments faced with the prospect of a shrinking workforce to support swelling numbers of retirees [3]. In response to these worries, there has been an increasing interest in what is called "healthy aging," the concept that ill-health is not an inevitable outcome of advancing years and that, with the appropriate interventions, older people can remain healthy and active for far longer than has been the case in the past. It has been observed that many older people would prefer to remain employed, if their health permits it, and the data is generally supportive of the idea that engagement in the workforce may offer an individual both health and financial benefits [4]. Thus, healthy aging potentially offers a solution to both the health-related and financial concerns associated with aging populations.

A major driver of the increase in life expectancy has been the reduction of infectious disease as a major cause of mortality and morbidity [5]. Vaccination has been a crucial part of this improvement, by reducing mortality directly due to infectious disease and from complications [6]. Today, only infectious respiratory diseases (such as influenza and pneumonia) remain a frequent cause of adult mortality [7], and the mortality figures are dominated by so-called non-communicable diseases (NCDs) which have their highest incidence in older adults [5]. This change in the burden of disease is not confined to the wealthiest countries; indeed, it is already visible in all but the poorest nations [8]. Among those $>60$ years of age, NCDs already account for $>90 \%$ of the global disease burden. By 2030, it is estimated that even in low-income countries it will be over $50 \%$, thus far outpacing the burden due to infectious diseases [8]. All of this would imply that vaccination will play a minor part in attempts to improve healthy aging, and this perception is perhaps reflected in the very low vaccine coverage rates in most countries [9]. However, a growing body of clinical evidence suggests that this perception is misguided, as some vaccine-preventable diseases (VPDs) are clearly implicated in the poor outcomes associated with major NCDs such as ischaemic heart disease, stroke, and some forms of dementia [10-13]. Supporting these findings, controlled trials have shown that vaccination against VPDs such as influenza can reduce the overall risk of hospitalization and death by $30-50 \%$ in older populations with chronic comorbid NCDs, making vaccination a potent tool for improving the overall health of older adults [14-16] (Table 1: summary). In this review, we indicate some of the ways that current health policies undervalue adult vaccination as a preventive tool and argue that a more personalized, risk-based approach to vaccination ("P4 Medicine" or "predictive, preventive, personalized, and participatory medicine" [17]) may be a useful complement to traditional public health campaigns.

\section{Chronic NCDs: Prevention Is Key}

The NCDs that are responsible for the bulk of morbidity, disability, and mortality in older adults are all chronic conditions: ischaemic heart disease, stroke, chronic obstructive pulmonary disease, cancer of the trachea, bronchus and lung, diabetes, Alzheimer disease and other dementias, diabetes mellitus, and liver and stomach cancer [5]. The key to reducing the burden of these diseases is prevention, which needs to start long before symptoms become noticeable. Once a patient has a condition such as ischaemic heart disease, diabetes, or lung cancer, the disease can be treated but it is rarely, if ever, possible for the patient to fully revert to the state of health that existed before the disease developed. A parallel can be drawn between these diseases and the process of aging itself. In both cases, illness results from the accumulated damage from a series of physiological insults often acquired over many years, and the ability to maintain former levels of 
Table 1. Non-communicable diseases for which immunization against vaccine-preventable disease may improve overall health outcomes

\begin{tabular}{ll}
\hline Non-communicable disease & Effect of vaccination (not an exhaustive list of available evidence) \\
\hline $\begin{array}{l}\text { Alzheimer's disease/ } \\
\text { other forms of dementia }\end{array}$ & $\begin{array}{l}\text { Influenza vaccination of patients }>60 \text { years of age with CKD (who are at an elevated risk of } \\
\text { dementia) reduced the risk of dementia by 36\% [37] }\end{array}$ \\
\hline Stroke & $\begin{array}{l}\text { In 1 US study, zoster vaccination reduced the risk of stroke by approximately 50\% in } \\
\text { vaccinated adults aged 65-69 years [31]. Influenza vaccination appears to reduce the overall } \\
\text { risk of ischaemic stroke by 13\% in adults, although the beneficial effect varies widely in } \\
\text { different study populations [33] }\end{array}$ \\
\hline $\begin{array}{l}\text { Cardiovascular disease, including } \\
\text { primary cardiac arrest, myocardial } \\
\text { infarct, ischaemic heart disease, } \\
\text { and acute coronary syndrome }\end{array}$ & $\begin{array}{l}\text { Meta-analyses suggested an overall reduction of 56\% in the risk of cardiovascular mortality } \\
\text { among adults vaccinated against seasonal influenza [33]. They also indicated a 17\% reduction } \\
\text { in the risk of acute coronary syndromes in patients }>65 \text { years who received pneumococcal }\end{array}$ \\
\hline Type 1 diabetes & $\begin{array}{l}\text { While direct cause and effect remain controversial, studies in the USA [62] and Australia [63] } \\
\text { suggested a decline of 37 and 15\%, respectively, in the risk of developing type 1 diabetes in } \\
\text { rotavirus-vaccinated children in the period immediately after vaccination. A Finnish study [64] } \\
\text { found a smaller, non-significant reduction }\end{array}$ \\
\hline
\end{tabular}

It should be noted that there is substantial variation in the magnitude of the protective effect of vaccination reported against noncommunicable disease, depending on the type of study and the end point, the patient's age and comorbidities, and (for diseases like influenza), the seasonal severity of the disease. However, for the non-communicable diseases listed here, there is a strong and consistent trend towards protection in the available data.

health (often called "resilience") becomes reduced over time. There are other parallels as well: many of these diseases, in addition to the onset of age-related impairments, share specific risk activities, such as low levels of physical activity, smoking and poor nutrition, and clinical markers such as high blood sugar, dyslipidemia and some degree of immune dysfunction [18]. In particular, the bias towards inflammatory responses that develops with age (dubbed "inflammaging") has been suggested not just as a basis for increased susceptibility to disease in later life, but also as a primary mechanism driving aging itself [18]. Acute infections can act as repeated extrinsic triggers for inflammation, at any time of life, while chronic infections may contribute to inflammation over time. From a biological viewpoint, aging can be seen as the sum of all the changes to a person's underlying biological state over time, rather than being directly linked to the number of years they have attained. While some of this individual variation can be traced to inherent factors (e.g., genetic polymorphisms) and environmental factors (e.g., pollution), there is no doubt that preventative actions such as improved diet, exercise, smoking avoidance (or cessation), etc. can reduce the risk of developing many chronic diseases and also increase the number of years spent in good health $[19,20]$. From an individual's point of view, most of these interventions are either low-cost (e.g., exer- cise) or even cost-saving (e.g., smoking avoidance). However, preventative actions of this nature require the active participation of the individual, since they involve changes in behavioural patterns that are then sustained over a period of years or decades. Thus, while preventive medicine may be simple in theory, in practice considerable resources for education to encourage healthy behaviour are often required [21]. Furthermore, despite the potential benefits, spending on preventive medicine is typically a very small part of most health budgets. Addressing this requires recognizing that this is a multi-faceted problem and that the individual root causes need to be identified and addressed appropriately throughout life. The second step is to build health care systems where the preventative approach is incorporated at every stage.

\section{Building a New Treatment Paradigm: P4 Medicine}

While progress has been made in reducing the incidence of some chronic NCDs, the World Health Organization (WHO) concluded more than a decade ago that current health care models with their focus on acute care are failing to adequately address the rising burden of NCDs [22]. In the intervening years, recognition that a patient-centric approach may offer superior outcomes to 


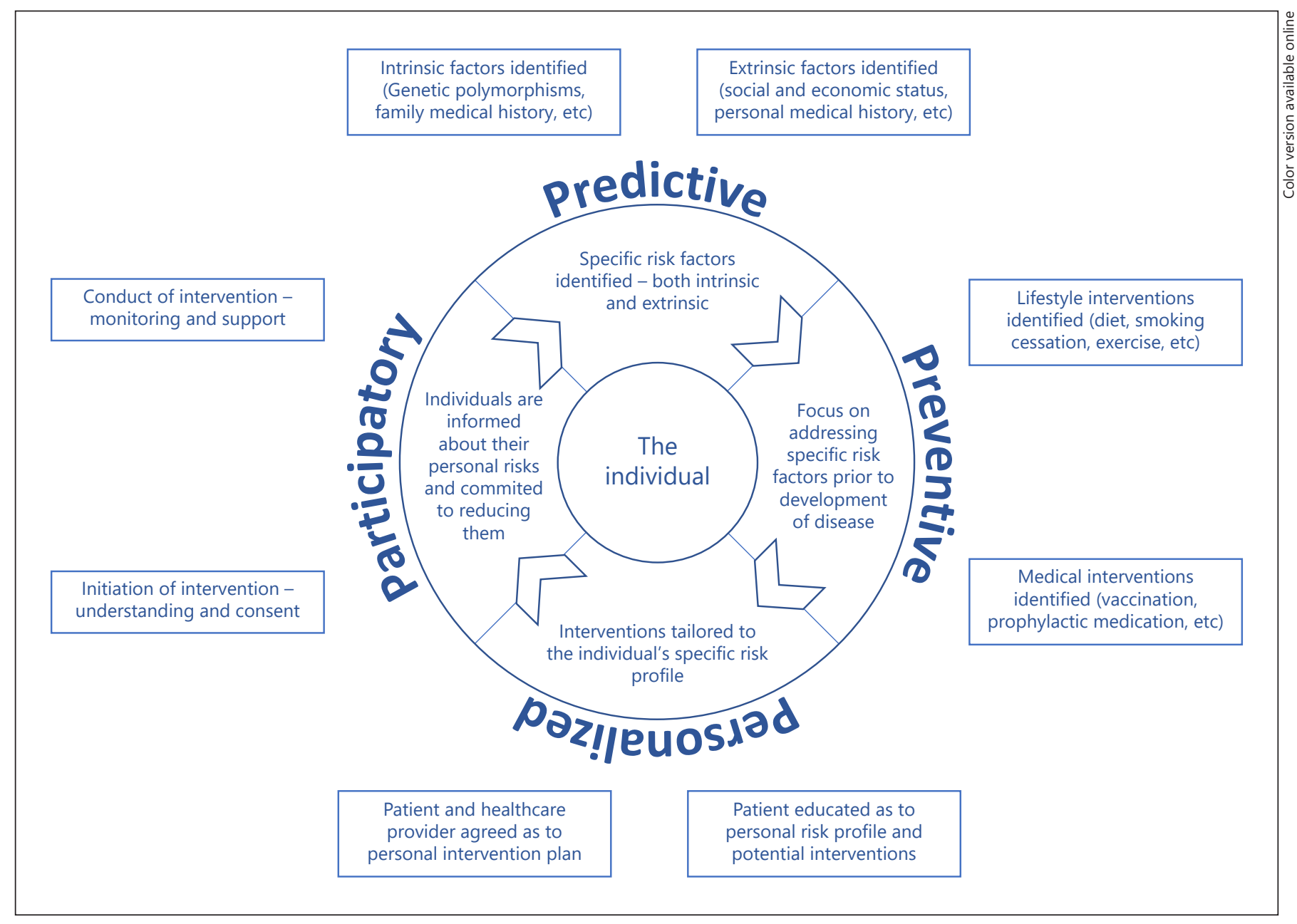

Fig. 1. P4 Medicine: predictive, preventive, personalized, and participatory (and iterative). As people age, their immunological capacity, risk factors, social and economic status, diet, and physical condition all change. In addition, there are broad differences in exposure to environmental and microbial agents. Even their ge-

a one-size-fits-all approach has crystallized into what is now often called "P4 Medicine," a contraction of the phrase "predictive, preventive, personalized, and participatory medicine" [17]. Though sometimes described as a whole new approach to medicine, $\mathrm{P} 4$ medicine can also be viewed as the natural extension of earliest practice: the Hippocratic school of medicine placed emphasis on the prevention of illness as well as its treatment, and urged practitioners to consider the social and physical environment along with a patient's symptoms when making a diagnosis [23]. While few physicians would disagree with this approach, putting it into practice has not always been easy. Today, however, access to vastly greater amounts of data, improved analytic tools to make sense

Older Adult Vaccination: From Reactive to Proactive netic make-up may be altered over time by epigenetic modification. Just as every individual is different, so too, the individual is different in infancy, childhood, adulthood, and old age. Therefore, reviewing and updating an individual's risk profile is necessary throughout life.

of the data, and a growing desire among consumers for "ownership" of their own health data are changing this picture. Taken together, these trends mean that, in the very near future, patients and their physicians are likely to have the ability to assemble a vastly more complete analysis of an individual's medical history than was previously possible. This will include not just their clinical history, but also the potential effects of their genome, epigenome, microbiome, and current health on proposed treatments. This is an iterative process; as a person ages, their risk factors, potential exposures, social and economic status, diet, and physical condition will all change, so reviewing and updating their risk profile is necessary (Fig. 1). 
The immune system changes throughout life and genomic screening has already identified a relatively restricted panel of pathogen-responsive (and pathogenspecific) genes that appears to consistently change with aging [24]. The focus of this data-driven approach to medicine is different from the current approach: instead of trying to match current symptoms to a specific diagnosis and then finding a treatment that will ameliorate those symptoms, the goal is to develop a picture of what "health" looks like for a particular individual in order to try and prevent or delay the development of symptomatic illness as far as possible. The first steps in this direction are already in evidence, with (for example) screening programs to attempt to catch cancers in their earliest (and most easily treated) stages, chemoprophylaxis to reduce the risk of myocardial infarction or hyperlipidemia, etc. [21]. These activities need to be carried out in the context of successful public health education campaigns, e.g., decreasing the incidence of lung cancer and cardiovascular disease by reducing smoking. The promise of the $\mathrm{P} 4$ medicine approach is that by personalizing the risk and the treatment of identifiable risks, physicians may be able to identify high-risk individuals, while the perception that this is "their personal treatment" may motivate individuals to be compliant with preventative behaviours.

\section{Vaccination as a Tool for Maintaining Wellness}

Traditionally, the benchmark for vaccination has been the population-level reduction in the incidence of acute infections, rather than using vaccination as a tool to promote wellness or overall health. The concept of life-course vaccination [25] is based on the recognition that, due to an age-related decline in immunity, vaccination in later life is unlikely to be completely effective at reducing the health burden of VPDs, and that effective immunity in later life builds on the immunity developed earlier in life. There is already good data available to support that adult mortality and morbidity can be greatly improved by childhood vaccination programs both through "herd" immunity, or by preventing childhood infections such as varicella or hepatitis that can cause serious disease in later life $[21,26]$. Beyond this, however, vaccination programs have been associated with decreases in mortality beyond that attributable to the specific pathogen targeted, presumably because the loss in resilience caused by infection renders those infected more susceptible to illness or death from other causes [27]. For this reason, it has been said that a good adult vaccination program starts with a good childhood vaccination program [28].

The benefits of preventing infectious disease can extend far beyond those which can be attributed to the acute illness associated with the pathogens. For example, while zoster and post-herpetic neuralgia are, in themselves, sufficiently serious to warrant vaccination in older adults, the link between the reactivation of herpes zoster and some NCDs may actually have more profound health consequences. It is estimated that reactivation of latent herpes zoster infections may increase the risk of stroke by as much as $50 \%$ in the month immediately after reactivation, with the elevated risk potentially persisting for months [10]. A meta-analysis of published studies suggested that reactivation of herpes zoster increases the risk of a cerebrovascular or cardiovascular event by $20-40 \%$ and $10-30 \%$, respectively, in the year after onset $[29,30]$, and that vaccination may approximately halve this risk in adults aged 65-69 years [31]. The consistent temporal association of zoster and an elevated risk of developing cardiac or cerebrovascular disease, together with the observation that anti-viral therapy or vaccination appear to diminish risk, argue strongly for a causal effect [30].

A similar pattern of elevated risk for cardiac and cerebrovascular events can be observed for influenza. While the severity of the influenza season is predictive of subsequent rates of hospitalization and mortality for cardiovascular disease in older adults, vaccination appears to reduce this risk [11,32]. There is good evidence to suggest that vaccination may be protective against myocardial infarction, and some suggesting it can offer protection against stroke, heart failure, and ischaemic heart disease (at least for patients with comorbidities) [33, 34].

A recent national-level analysis of heart failure patients from Denmark found that influenza vaccination reduced both all-cause mortality and mortality from cardiovascular events by $18 \%$ after adjustment for comorbidities and socioeconomic status [35]. In addition, more frequent and consistent vaccination was associated with lower mortality [35]. A prospective study of older adults (aged $\geq 65$ years) with comorbidities demonstrated that participants receiving influenza or influenza and pneumococcal (polysaccharide) vaccines had significantly reduced admissions to the coronary unit and intensive care unit [14]. Over the 18-month study period, participants receiving both vaccines saw significant reductions in coronary and intensive care admissions of 41 and 55\%, respectively [14]. Population-based cohort studies on older adults ( $>55$ years) with comorbidities that put them at risk for cardiovascular disease found a clear association 
between decreased risk of acute coronary syndrome hospitalization and influenza vaccination $[15,16]$. The vaccine caused an overall reduction in hospital admissions of $56 \%$ for acute coronary syndromes for patients with chronic obstructive pulmonary disease, and $65 \%$ for patients with chronic kidney disease (CKD) $[15,16]$.

Intriguingly, a separate analysis by the same group found that influenza vaccination of patients with CKD, itself thought to be a risk factor for cognitive decline and dementia [36], also reduced the risk of dementia in these patients in a dose-dependent manner [37]. This suggests that prevention of influenza may also yield benefits for dementia, another leading cause of NCDs in older adults. While an infectious aetiology for Alzheimer's disease has long been postulated, evidence of a link has been weak [38]. However, observational studies suggesting that anti-viral treatment or vaccination can reduce dementia risk support the idea that common infections may be a risk factor for dementia, even if they are not the actual cause $[12,37,39]$. What ties these apparently disparate diseases together is the postulated mechanism of action: "Inflammaging" [18], i.e., the persistent activation of inflammatory immune responses at least partially due to acute infection or reactivation of a latent infection, leading to immunopathology that accumulates over time [34, 38]. Taken together, these studies provide a substantial body of evidence that vaccination can play a major role in reducing morbidity and mortality from NCDs as well as infectious diseases in older adults.

\section{Integrating Vaccination into a P4 Medicine Strategy}

At first glance, vaccination does not seem a natural fit for P4 medicine: while vaccination is by nature preventative, perhaps more than any other medical intervention it has traditionally been implemented at a population level, with essentially identical products administered to millions or tens of millions of individuals, regardless of their individual risk. However, we would argue that just as with, for example, smoking cessation, public health campaigns and a focus on wellness at the level of the individual can be useful complementary approaches, especially when it comes to vaccinating older adults with comorbidities or frailty, for whom the risks are significant and better defined. A move towards "personalizing" vaccination recommendations does not mean abandoning the universal vaccination recommendations. The biggest known risk factor for many VPDs is simply a lack of immunity, so maintaining high levels of coverage will remain a priority. Unfortunately, current adult vaccination programs struggle to deliver the

Older Adult Vaccination: From Reactive to Proactive coverage levels that are routinely provided by paediatric vaccination programs, resulting in coverage well below the thresholds needed for effective herd immunity against most VPDs [9]. Increased uptake at the individual level means better coverage at the population level, and, potentially, improved outcomes for the whole community.

The question as to why adult vaccination rates rarely reach the necessary level of uptake is a complex one and is beyond the scope of this review. A lack of prioritization by health care authorities may play a role. For example, even in regions such as Europe, it has taken far longer to implement adult vaccine recommendations, with many countries still lacking comprehensive national policies for adult vaccination [9]. The lack of infrastructure for adult vaccination may also be a problem: paediatric vaccination is built on a long-standing tradition of prenatal and infant health visits, and health care utilization by the mother has long been known to correlate with vaccine uptake [40,41]. But a low engagement of adults with vaccination appears to be major factor. The lack of a doctor's recommendation (which may be in part due to the lack of structured vaccination or wellness visits for most adults) and the public perception that vaccination is not important for healthy adults are among the commonest reasons for not vaccinating [42]. This is further complicated by the fact that adult immunization programs are usually not universal, but instead based on broad risk groups identified by age, pre-existing comorbidity, immune deficiency, pregnancy, etc. [43].

P4 medicine offers the potential to better define those who are most at risk for adverse events from vaccination, or individuals at risk for certain conditions (including NCDs) who will derive the most benefit from vaccination. Early studies in using computer analyses of health records to identify high-risk patients and target them for vaccination increased coverage, including those who had not previously been identified as at risk [44], and digital outreach generally appears to improve vaccine uptake [45]. What P4 medicine adds to this approach is the possibility to make the risks and benefits personal, rather than presenting them as unavoidable consequences of being a certain age and thus as potentially actionable.

One size does not always fit all (Fig. 2). This is not purely a question of acceptance by patients. It also involves the acceptance by physicians that vaccination is an important part of an individual patient's care and its implementation. For example, a step as simple as adding a check on vaccination status and needs to the patient's visit can significantly improve uptake [46]. The WHO has included access to vaccines as part of their focus on "older person-centred and integrated care" in a recent policy 


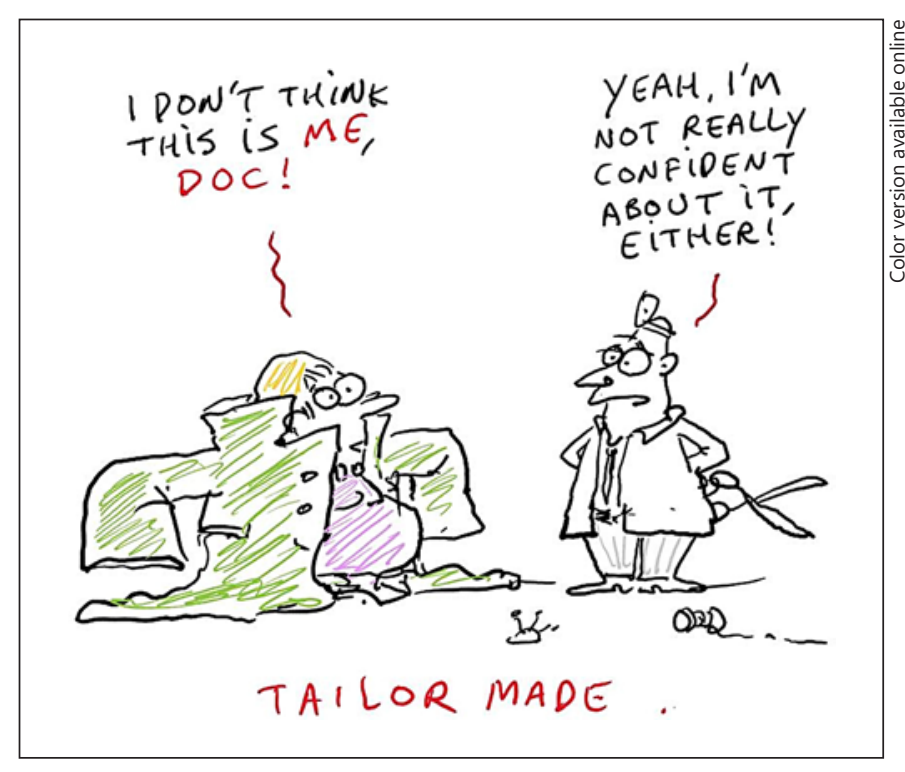

Fig. 2. Medical intervention is not "one-size-fits-all". Figure reused with permission from ComicHouse ${ }^{\circledR}$ (Floris Oudshoorn/ComicHouse.nl).

recommendation as well as observing that an individual's functional capacity may be a more useful indicator of the need for vaccination than their chronological age [2, 47].

There are signs that this may be changing. While recommendations for managing cardiovascular patients previously recognised the link between respiratory infection and acute myocardial infarction, and recommended vaccination, this has not always been accorded priority [48]. While there is a role for all health care providers that work with older adults to be prepared to discuss vaccination as part of their health maintenance activities, the needed training and support is not readily available [43]. However, more recent recommendations have started to take an integrated approach, emphasizing influenza and zoster vaccination as an important component of ongoing patient care for cardiovascular disease [49]. Moreover, vaccination is increasingly placed in the context of preventative activities against cardiovascular disease that start in childhood and continue throughout life, but that also take into account factors such as familial risk, personal exposure history, comorbidities, and obesity [49]. These are nonetheless small, initial steps.

While the preventative role of vaccination in enhancing healthy aging is becoming better appreciated, to fully implement vaccination into the $\mathrm{P} 4$ medicine paradigm requires attention be paid to the "predictive" and "personalized" aspects. To a certain extent, this is already done by assigning people to risk groups for whom specific vaccinations are indicated or contra-indicated. Likewise, vaccina-
Box 1. Can a P4 approach really improve vaccine uptake and overall health in adults as a complement to public health interventions?

While supporting data are not conclusive, there are reasons to believe that "personalizing" vaccine recommendations may help improve compliance with universal vaccination recommendations. The first reason is our experience with existing public health campaigns, where a desire to protect/ improve personal health appears to be a driver of compliance with recommendations [21], e.g., cervical cancer screening (where, typically, participation in screening programs far exceeds the vaccination rate in adults [64] despite the fact that it requires more effort on the part of the patient). The second reason is the existing evidence that health-seeking behaviour is, in many cases, associated with improved vaccination coverage.

An indication of the potential of vaccination to improve health outcomes is the way that we currently deal with the prevention of cardiac disease. Lipid testing and a discussion about lifestyle during consultations are both used to identify risk which is the basis for prescribing cholesterol-lowering medicines which have as their goal a reduced risk of future cardiac disease. A great deal of effort is expended on interventions such as statins or smoking cessation despite observations suggesting that these have significantly less preventative efficacy against cardiac diseases than influenza vaccination [11, 33-35, 65]. Inexplicably, despite the evidence that influenza vaccination can reduce the risk of cardiac disease [11,33-35], it is not typically prescribed for adults $<65$ years until after a cardiac event. Drug treatment for hyperlipidaemia is prescribed on the basis of risk [48, 49], not medical history, with the goal of preventing a cardiac event occurring at all. By using a person's risk (identified by a P4 approach) rather than their medical history as a guide to recommending vaccination, a substantial disease burden could potentially be avoided.

tion against diseases such as chronic hepatitis infection or cervical cancer, that will manifest primarily as severe disease much later in life, are widespread. These recommendations are driven by a recognition of future risk at a population level, so this does not require a paradigm shift. What P4 medicine suggests is a better evidence-driven understanding of individual risk. To take a simple example, some vaccines, such as those against influenza, are often recommended for a whole age cohort, e.g., people aged $\geq 65$ years. This is based on the observed elevated risk for severe outcomes in this cohort as a whole. However, immunocompetence and biological age do not always correlate with chronological age [21]. Some individuals (e.g., those with an elevated risk of developing cardiovascular disease) may benefit from vaccination earlier in life [47]. The aim is to avert a cardiac event by vaccination, rather than assigning them to a high-risk group after the cardiac event and then 
recommending vaccination (Box 1). In short, though the goal of current recommendations is preventative, the predictive aspect is cohort-based, not individual-based, and prevention is based on reducing acute cases of VPD, rather than protecting overall health. To build an adult vaccination programme around defined, preventable individual risks (both known and as yet undiscovered) requires a comprehensive approach to data-gathering. Leroy Hood, who coined the phrase "P4 Medicine," calls this "a dense dynamic data cloud," i.e., the monitoring of an individual's health status that spans their genomics, clinical blood chemistry, and gut microbiome as well as data such as weight, diet, exercise, etc., and which, crucially, is updated over time to reflect an individual's changing biology [17, 50]. The goal of such an analysis is not to detect disease for treatment, but to optimize wellness and avoid disease as much as possible $[17,50]$. By tracking biomarkers, in large numbers of healthy people, the ultimate goal is to identify patterns that indicate a wellness-to-disease transition, allowing for early intervention including, but not limited to, vaccination [50]. While the levels of data collection needed to draw these correlations are currently not standard, nothing described here is beyond the reach of current technology. It is more of a practical/economical challenge than a technological one.

The final part of the puzzle is the participatory aspect which applies to the patients themselves; all the data collected is useless if individuals do not consent to the intervention. This is particularly true of vaccination, where the intervention is intended to prevent future risk rather than to reduce current symptoms. The first step in this direction is education. A poor understanding of vaccination recommendations is linked to poor vaccine uptake, even in health care systems with good resources [51]. By identifying and personalizing the risk of disease and the benefits of vaccination for individual patients and involving them more in the discussion (e.g., by including a more detailed analysis of lifestyle and familial factors), it is hoped that they will be more motivated to comply.

\section{Discussion}

Little of what has been discussed here is entirely new conceptually. The prevention of disease and targeting interventions to those who will reap the greatest benefit are uncontroversial aspects of modern medicine. What is new is the concept of gathering and analyzing data at a level that was not technically possible in the past, and then applying the analysis to delivering medical advice for the

Older Adult Vaccination: From Reactive to Proactive individual patient, what Leroy Hood calls "actionable possibilities" [50]. Several changes have now made this possible, and, given the recent trends, even likely. The first is the rapidly increasing ability of machine-learning approaches to swiftly analyze very large datasets and extract insights from them. The second is the trend towards comprehensive electronic health records [52], which makes an individual's prior health history accessible as well as, potentially, the health history of other family members. The third is the increasing number of data sources available, ranging from whole-genome sequencing and blood metabolite analysis, to the proliferation of health and exercise apps already used by individuals themselves to monitor diet, exercise, sleep patterns, blood pressure, etc.

The cost of gathering this data has decreased dramatically in recent years. In particular, the ubiquity of mobile phones and the vast number of health-related apps and devices means that it is feasible to cheaply collect longitudinal, individualized data on a scale that would have been inconceivable even a decade ago $[53,54]$. The proliferation of health apps also signals an interest among the general public to participate in the care of their own health, which bodes well for adopting preventative measures. The issue, therefore, is not so much obtaining the data, but aggregating it into formats that can crosstalk with each other and be used for analysis in toto [55]. We acknowledge that the aggregation of data may bring some ethical issues that require consideration, but it will allow health care professionals to make individual informed recommendations on "actionable possibilities" such as vaccination, diet changes, physical exercise, or medical intervention, but also to check compliance with existing treatment recommendations and guard against potentially negative interactions with existing medications, genetic predispositions, or comorbidities [56]. In the longer term, a better understanding of the interplay of immunosenescence, general health, and vaccination may also guide the development of more effective vaccines for older patients [57].

It is unclear what the costs will be of assembling such a package of data and providing effective coaching about what the data means for the individual. Start-up companies which are operating in this arena in the USA have shown that it is technologically feasible [58] but have struggled with the economies of scale needed to make it commercially viable, so whether national health authorities could adopt this approach more successfully remains unclear. However, like most technological solutions, it can reasonably be expected that costs will fall significant- 
ly with time and with adoption at scale. In addition, such costs need to be set against the substantial direct saving that will potentially be achieved. Some vaccination studies have already reported a reduction in hospitalization rates greatly exceeding $50 \%$ in older patients with comorbidities $[15,16]$.

There are also the issues surrounding the aggregation and centralization of something as sensitive as health data which will need to be addressed in a transparent manner before public (or indeed medical) acceptance can be assured [59]. While the boom in health apps, the expansion of companies such as 23andMe (Mountain View, CA, USA) that offer health recommendations based on genetic markers, and the increasing interest in well-being suggest that younger adults are, to some extent, already primed for this shift, convincing the older generation may take time.

Finally, there is the issue of implementation. Medicine is traditionally conservative in its adoption of new practices, with good reason since the health of patients is at stake. Smaller-scale integration of electronic records can be shown to improve both patients' experience and the clinical workflow $[60,61]$ but implementation on a larger scale is likely to take time. Nonetheless, the pressures of an aging population and the failure of current medical paradigms to fully address the issue of healthy aging mean that change is desirable, and a data-driven move towards promoting wellness rather than treating illness is a response that is feasible.

\section{Acknowledgement}

We would like to thank the Business \& Decision Life Sciences platform for editorial assistance, manuscript coordination, and for design support for the digital animations on behalf of GSK. Carole Desiron coordinated manuscript development and editorial support. The authors would also like to acknowledge the VITAL project supported by the Innovative Medicines Initiative 2 Joint Undertaking (JU) under grant agreement No. 806776. The JU receives support from the European Union's Horizon 2020 research and innovation programme and EFPIA.

\section{Disclosure Statement}

T.M.D., G.D.G., and A.D.P. are employees of and hold shares in the GSK group of companies. G.D.G. is member of the editorial board of Gerontology but was not involved in the peer-review process of the article. J.-P.M. was member of the scientific board of the Institute Servier (2002-2018) and an advisor for Pfizer (2016) and Merck (2016) outside of this work.

\section{Funding Sources}

GlaxoSmithKline Biologicals S.A. funded this work and all costs related to the development of the review article.

\section{Author Contributions}

All authors reviewed the literature, provided substantial input, contributed to writing, and reviewed the paper. T.M.D. led the manuscript development. All authors gave their final approval and are accountable for all aspects of the work.

\section{References}

1 Oeppen J, Vaupel JW. Demography. Broken limits to life expectancy. Science. 2002 May; 296(5570):1029-31.

2 Beard JR, Officer A, de Carvalho IA, Sadana R, Pot AM, Michel JP, et al. The World report on ageing and health: a policy framework for healthy ageing. Lancet. 2016 May;387(10033): 2145-54.

3 World Health Organization Workforce 2030 [Internet]. Global strategy on human resources for health. WHO Library. 2016 [cited 2019 Apr 9]. Available from: https://apps.who. int/iris/bitstream/handle/10665/250368/ 9789241511131-eng.pdf;jsessionid=2BA9592 C586D2A63F871CAC1EA3630FF?sequen$\mathrm{ce}=1$

4 Naegle G, Bauknecht J. Extending working lives. The Future of Ageing in Europe: Making an Asset of Longevity. Singapore, Singapore: Palgrave Macmillan; 2019. https://doi. org/10.1007/978-981-13-1417-9_5.

5 Jones DS, Podolsky SH, Greene JA. The burden of disease and the changing task of medicine. N Engl J Med. 2012 Jun;366(25):2333-8.
6 Centers for Disease Control and Prevention (CDC). Ten great public health achievements - United States, 1900-1999. MMWR Morb Mortal Wkly Rep. 1999 Apr;48(12): 241-3.

7 Gavazzi G, Krause KH. Ageing and infection. Lancet Infect Dis. 2002 Nov;2(11):659-66.

8 World Health Organization [Internet]. Projections of mortality and causes of death, 2016 to 2060. WHO Library. 2016 [cited 2019 Apr 9]. Available from: https://www.who.int/ healthinfo/global_burden_disease/projections/en/

9 Sheikh S, Biundo E, Courcier S, Damm O, Launay O, Maes E, et al. A report on the status of vaccination in Europe. Vaccine. 2018 Aug; 36(33):4979-92.

10 Forbes HJ, Williamson E, Benjamin L, Breuer J, Brown MM, Langan SM, et al. Association of herpesviruses and stroke: systematic review and meta-analysis. PLoS One. 2018 Nov; 13(11):e0206163.

11 Warren-Gash C, Smeeth L, Hayward AC. Influenza as a trigger for acute myocardial in- farction or death from cardiovascular disease: a systematic review. Lancet Infect Dis. 2009 Oct;9(10):601-10.

12 Itzhaki RF. Corroboration of a Major Role for Herpes Simplex Virus Type 1 in Alzheimer's Disease. Front Aging Neurosci. 2018 Oct;10: 324.

13 Lövheim H, Gilthorpe J, Adolfsson R, Nilsson LG, Elgh F. Reactivated herpes simplex infection increases the risk of Alzheimer's disease. Alzheimers Dement. 2015 Jun;11(6):593-9.

14 Hung IF, Leung AY, Chu DW, Leung D, Cheung T, Chan CK, et al. Prevention of acute myocardial infarction and stroke among elderly persons by dual pneumococcal and influenza vaccination: a prospective cohort study. Clin Infect Dis. 2010 Nov;51(9):1007-16.

15 Sung LC, Chen CI, Fang YA, Lai CH, Hsu YP, Cheng $\mathrm{TH}$, et al. Influenza vaccination reduces hospitalization for acute coronary syndrome in elderly patients with chronic obstructive pulmonary disease: a populationbased cohort study. Vaccine. 2014 Jun;32(30): 3843-9. 
16 Chen CI, Kao PF, Wu MY, Fang YA, Miser JS, Liu JC, et al. Influenza Vaccination is Associated with Lower Risk of Acute Coronary Syndrome in Elderly Patients with Chronic Kidney Disease. Medicine (Baltimore). 2016 Feb; 95(5): 2588.

17 Hood L, Flores M. A personal view on systems medicine and the emergence of proactive $\mathrm{P} 4$ medicine: predictive, preventive, personalized and participatory. N Biotechnol. 2012 Sep;29(6):613-24.

18 Franceschi C, Garagnani P, Vitale G, Capri M, Salvioli S. Inflammaging and 'Garb-aging'. Trends Endocrinol Metab. 2017 Mar;28(3): 199-212.

19 Turner JE, Lira VA, Brum PC. New Insights into the Benefits of Physical Activity and Exercise for Aging and Chronic Disease. Oxid Med Cell Longev. 2017;2017:2503767.

20 Allison RL. Back to Basics: The Effect of Healthy Diet and Exercise on Chronic Disease Management. South Dakota Med. 2017;5:1018.

21 Doherty TM, Del Giudice G, Maggi S. Adult vaccination as part of a healthy lifestyle: moving from medical intervention to health promotion. Ann Med. 2019 Mar;51(2):12840.

22 Pruitt S, Annandale S, Epping-Jordan J, Fernandez Diaz J, Khan M, Kisa A, et al. Innovative care for chronic conditions: building blocks for action. A global report [Internet]. WHO Library. 2002 [cited 2019 Apr 19]. Available from: https://www.who.int/chp/ knowledge/publications/icccreport/en/

23 Kleisiaris CF, Sfakianakis C, Papathanasiou IV. Health care practices in ancient Greece: the Hippocratic ideal. J Med Ethics Hist Med. 2014 Mar; 7:6

24 Piasecka B, Duffy D, Urrutia A, Quach H, Patin E, Posseme C, et al.; Milieu Intérieur Consortium. Distinctive roles of age, sex, and genetics in shaping transcriptional variation of human immune responses to microbial challenges. Proc Natl Acad Sci USA. 2018 Jan; 115(3):E488-97.

25 Gusmano MK, Michel JP. Life course vaccination and healthy aging. Aging Clin Exp Res. 2009 Jun;21(3):258-63.

26 Kim TH, Johnstone J, Loeb M. Vaccine herd effect. Scand J Infect Dis. 2011 Sep;43(9):6839.

27 Bonanni P. Demographic impact of vaccination: a review. Vaccine. 1999 Oct;17 Suppl 3:S120-5.

28 Lang PO, Govind S, Michel JP, Aspinall R, Mitchell WA. Immunosenescence: implications for vaccination programmes in adults. Maturitas. 2011 Apr;68(4):322-30.

29 Erskine N, Tran H, Levin L, Ulbricht C, Fingeroth J, Kiefe C, et al. A systematic review and meta-analysis on herpes zoster and the risk of cardiac and cerebrovascular events. PLoS One. 2017 Jul;12(7):e0181565.

30 Zhang Y, Luo G, Huang Y, Yu Q, Wang L, Li K. Risk of Stroke/Transient Ischemic Attack or Myocardial Infarction with Herpes Zoster:
A Systematic Review and Meta-Analysis. J Stroke Cerebrovasc Dis. 2017 Aug;26(8): 1807-16.

31 Klaric JS, Beltran TA, McClenathan BM. An Association Between Herpes Zoster Vaccination and Stroke Reduction Among Elderly Individuals. Mil Med. 2019 Mar;184 Suppl 1: 126-32.

32 Nguyen JL, Yang W, Ito K, Matte TD, Shaman J, Kinney PL. Seasonal Influenza Infections and Cardiovascular Disease Mortality. JAMA Cardiol. 2016 Jun; 1(3):274-81.

33 Fountoulaki K, Tsiodras S, Polyzogopoulou E, Olympios C, Parissis J. Beneficial Effects of Vaccination on Cardiovascular Events: Myocardial Infarction, Stroke, Heart Failure. Cardiology. 2018;141(2):98-106.

34 Meyers DG. Myocardial infarction, stroke, and sudden cardiac death may be prevented by influenza vaccination. Curr Atheroscler Rep. 2003 Mar;5(2):146-9.

35 Modin D, Jørgensen ME, Gislason G, Jensen JS, Køber L, Claggett B, et al. Influenza Vaccine in Heart Failure. Circulation. 2019 Jan; 139(5):575-86

36 Etgen T, Chonchol M, Förstl H, Sander D. Chronic kidney disease and cognitive impairment: a systematic review and meta-analysis. Am J Nephrol. 2012;35(5):474-82.

37 Liu JC, Hsu YP, Kao PF, Hao WR, Liu SH, Lin CF, et al. Influenza Vaccination Reduces Dementia Risk in Chronic Kidney Disease Patients: A Population-Based Cohort Study. Medicine (Baltimore). 2016 Mar; 95(9):e2868.

38 Wang Y, Ding L, Zhu Q, Shu M, Cai Q. Common Infections May Lead to Alzheimer's Disease. Virol Sin. 2018 Oct;33(5):456-8.

39 Devanand DP. Viral Hypothesis and Antiviral Treatment in Alzheimer's Disease. Curr Neurol Neurosci Rep. 2018 Jul;18(9):55.

40 Wiecha JM, Gann P. Does maternal prenatal care use predict infant immunization delay? Fam Med. 1994 Mar;26(3):172-8.

41 Kogan MD, Alexander GR, Jack BW, Allen MC. The association between adequacy of prenatal care utilization and subsequent pediatric care utilization in the United States. Pediatrics. 1998 Jul;102(1 Pt 1):25-30.

42 de Gomensoro E, Del Giudice G, Doherty TM. Challenges in adult vaccination. Ann Med. 2018 May;50(3):181-92.

43 Bonanni P, Bonaccorsi G, Lorini C, Santomauro F, Tiscione E, Boccalini S, et al. Focusing on the implementation of 21 st century vaccines for adults. Vaccine. 2018 Aug;36(36): 5358-65.

44 Hak E, van Essen GA, Stalman WA, de Melker RA. Improving influenza vaccination coverage among high-risk patients: a role for computer-supported prevention strategy? Fam Pract. 1998 Apr;15(2):13843.

45 Atkinson KM, Wilson K, Murphy MS, ElHalabi S, Kahale LA, Laflamme LL, et al. Effectiveness of digital technologies at improving vaccine uptake and series completion - $\mathrm{A}$ systematic review and meta-analysis of randomized controlled trials. Vaccine. 2019 May; 37(23):3050-60.

46 McKibben LJ, Stange PV, Sneller VP, Strikas RA, Rodewald LE. Use of standing orders programs to increase adult vaccination rates. MMWR Recomm Rep. 2000;49(Rr-1): 15-6.

47 Teresa Aguado M, Barratt J, Beard JR, Blomberg BB, Chen WH, Hickling J, et al. Report on WHO meeting on immunization in older adults: Geneva, Switzerland, 22-23 March 2017. Vaccine. 2018 Feb;36(7):921-31.

48 Piepoli MF, Hoes AW, Agewall S, Albus C, Brotons C, Catapano AL, et al.; ESC Scientific Document Group. 2016 European Guidelines on cardiovascular disease prevention in clinical practice: The Sixth Joint Task Force of the European Society of Cardiology and Other Societies on Cardiovascular Disease Prevention in Clinical Practice (constituted by representatives of 10 societies and by invited experts)Developed with the special contribution of the European Association for Cardiovascular Prevention \& Rehabilitation (EACPR). Eur Heart J. 2016 Aug;37(29): 2315-81.

49 Volpe M, Battistoni A, Gallo G, Rubattu S, Tocci G; Writing Committee; Scientific Societies. Executive Summary of the 2018 Joint Consensus Document on Cardiovascular Disease Prevention in Italy. High Blood Press Cardiovasc Prev. 2018 Sep;25(3):327-41.

50 Hood L. P4 Medicine and the Democratization of Health Care [Internet]. NEJM Catalyst. 2017 [cited 2019 Mar 29]. Available from: https://catalyst.nejm.org/p4-medicine-democratization-health/

51 Akmatov MK, Rübsamen N, Deyneko IV, Karch A, Mikolajczyk RT. Poor knowledge of vaccination recommendations and negative attitudes towards vaccinations are independently associated with poor vaccination uptake among adults - Findings of a populationbased panel study in Lower Saxony, Germany. Vaccine. 2018 Apr;36(18):2417-26.

52 Buntin MB, Burke MF, Hoaglin MC, Blumenthal $\mathrm{D}$. The benefits of health information technology: a review of the recent literature shows predominantly positive results. Health Aff (Millwood). 2011 Mar;30(3):464-71.

53 Gay V, Leijdekkers P. Bringing Health and Fitness Data Together for Connected Health Care: Mobile Apps as Enablers of Interoperability. J Med Internet Res. 2015 Nov; 17(11):e260.

54 Valderas JM, Kotzeva A, Espallargues M, Guyatt G, Ferrans CE, Halyard MY, et al. The impact of measuring patient-reported outcomes in clinical practice: a systematic review of the literature. Qual Life Res. 2008 Mar: 17(2):179-93.

55 Choi IY, Kim TM, Kim MS, Mun SK, Chung YJ. Perspectives on clinical informatics: integrating large-scale clinical, genomic, and health information for clinical care. Genomics Inform. 2013 Dec;11(4):186-90. 
56 Klann JG, McCoy AB, Wright A, Wattanasin N, Sittig DF, Murphy SN. Health care transformation through collaboration on opensource informatics projects: integrating a medical applications platform, research data repository, and patient summarization. Interact J Med Res. 2013 May;2(1):e11.

57 Crooke SN, Ovsyannikova IG, Poland GA, Kennedy RB. Immunosenescence: A systemslevel overview of immune cell biology and strategies for improving vaccine responses. Exp Gerontol. 2019 Sep; 124:110632.

58 Zubair N, Conomos MP, Hood L, Omenn GS, Price ND, Spring BJ, et al. Genetic Predisposition Impacts Clinical Changes in a Lifestyle Coaching Program. Sci Rep. 2019 May;9(1): 6805.
59 Andreu-Perez J, Poon CC, Merrifield RD, Wong ST, Yang GZ. Big data for health. IEEE J Biomed Health Inform. 2015 Jul;19(4): 1193-208.

60 Yoo S, Hwang H, Jheon S. Hospital information systems: experience at the fully digitized Seoul National University Bundang Hospital. J Thorac Dis. 2016 Aug;8(S8 Suppl 8):S63741.

61 Hochron S, Goldberg P. Driving physician adoption of mHeath solutions. Healthc Financ Manage. 2015 Feb;69(2):36-9.

62 Rogers MA, Basu T, Kim C. Lower Incidence Rate of Type 1 Diabetes after Receipt of the Rotavirus Vaccine in the United States, 2001-2017. Sci Rep. 2019 Jun;9(1): 7727.
63 Perrett KP, Jachno K, Nolan TM, Harrison LC. Association of Rotavirus Vaccination With the Incidence of Type 1 Diabetes in Children. JAMA Pediatr. 2019 Mar;173(3): 280-2.

64 Vaarala O, Jokinen J, Lahdenkari M, Leino T. Rotavirus Vaccination and the Risk of Celiac Disease or Type 1 Diabetes in Finnish Children at Early Life. Pediatr Infect Dis J. 2017 Jul;36(7):674-5.

65 Raina MacIntyre C, Menzies R, Kpozehouen E, Chapman M, Travaglia J, Woodward M, et al. Equity in disease prevention: vaccines for the older adults - a national workshop, Australia 2014. Vaccine. 2016 Nov;34(46):54639. 